\title{
Allergische Rhinitis: ARIA-Klassifizierung nicht ausreichend
}

\section{Die ARIA-Klassifizierung der allergischen Rhinitis basiert auf der Schwere und Dauer der nasalen Symptome. Bezieht man die entzündlichen Prozesse in der Nase mit ein, ergibt sich ein etwas modifiziertes Bild.}

$\mathrm{D}$ ie ARIA (Allergic Rhinitis and Its Impact on Asthma)-Klassifizierung unterscheidet einerseits intermittierende und persistierende, andererseits leichte und moderate bis schwere Symptome der Allergischen Rhinitis (AR). Ob sich eine solche Einteilung nach Schwere und Dauer der Symptome in den entzündlichen Prozessen der Nase widerspiegelt, untersuchten thailändische Allergologen. Sie nutzten dazu in ihrer Querschnitt-Studie die mukoziliäre Clearance-Zeit (MCCT) im Holzkohle-Saccharin-Test als messbare Größe der nasalen Entzündung.

Von 73 Patienten mit AR stuften die Autoren zwei Drittel $(67,1 \%)$ als moderat bis schwer betroffen ein. Die MCCT war am schlechtesten bei Patienten mit nach ARIA-Leitlinien mittelschwerer bis schwerer persistierender Symptomatik (14,2 min). Es folgten Patienten, mit mittelschweren bis schweren intermittierenden Symptomen (13,9 min), solche mit leichter persistierender Symptomatik (11,9 min) und solche mit leichten intermittierenden Symptomen (10,3 min).

In der Studie wurden die Schwere der nasalen Einzelsymptome in 3 Schwergraden erfasst $(1=$ mild, $2=$ moderat, $3=$ schwer) und daraus der Gesamtsymptomwert (TSS) gebildet. Der TSS korrelierte gut mit der MCCT der Nase ( $p<0,001)$, weniger gut und nicht statistisch signifikant allerdings mit der Zahl der Tage mit Symptomen $(\mathrm{p}=0,065)$. Die Clearance als Marker der nasalen Entzündung steht also in einem engen Zusammenhang mit der Schwere der Symptomatik, weniger aber mit der Dauer.

Fazit: Die MCCT zeigt eine deutliche Heterogenität innerhalb moderater bis schwerer AR-Symptome. Die Autoren raten daher, klarer zwischen diesem Symptomgrad zu unterscheiden und dadurch homogenere Patientengruppen zu erhalten, die von entsprechend angepassten Therapien profitieren könnten. $\mathbf{f k}$

Kirtsreesakul V et al. The correlation between nasal symptom and mucociliary clearance in allergic rhinitis.

Laryngoscope. 2009;119:1458-62 